\title{
CYTOTOXIC CONSTITUENT IN THE FRUIT PEEL OF Lansium domesticum
}

\author{
Q. Labibah ${ }^{1}$, K. N. W. Tun ${ }^{1,2}$, N. S. Aminah ${ }^{1,3,}$, A. N. Kristanti ${ }^{1,3}$, R. \\ Ramadhan $^{1}$, Y. Takaya ${ }^{4}$, C. A. C. Abdullah ${ }^{5,6,7}$ and M. J. Masarudin ${ }^{8}$ \\ ${ }^{1}$ Department of Chemistry, Faculty of Science and Technology, Universitas Airlangga, Komplek \\ Kampus C UNAIR, JL. Mulyorejo, Surabaya, 60115 Indonesia \\ ${ }^{2}$ Department of Chemistry, Pathein University, Pathein, Myanmar \\ ${ }^{3}$ Biotechnology of Tropical Medicinal Plants Research Group, Universitas Airlangga \\ ${ }^{4}$ Faculty of Pharmacy, Meijo University, 150 Yagotoyama, Tempaku, Nagoya, 468-8503 Japan \\ ${ }^{5}$ Department of Physics, Faculty of Science, University Putra Malaysia, 43400 UPM Serdang, \\ Selangor, Malaysia \\ ${ }^{6}$ Material Synthesis and Characterization Laboratory, Institute of Advanced Technology, \\ Universiti Putra Malaysia, 43400 UPM Serdang, Selangor, Malaysia \\ ${ }^{7}$ UPM- MAKNA Cancer Research Lab, Institute of Biosciences, Universiti Putra Malaysia, \\ 43400 UPM Serdang, Selangor, Malaysia \\ ${ }^{8}$ Department of Cell and Molecular Biology, Faculty of Biotechnology and Biomolecular \\ Sciences, UPM \\ ${ }^{\square}$ Corresponding Author: nanik-s-a@fst.unair.ac.id
}

\begin{abstract}
Phytochemical study of the hexane fruits peel extract of Lansium domesticum contributed to the discovery of onoceranoid-type triterpene, onoceradienedione 1. The structure of the isolated compound was done by advance spectroscopic techniques, comprising IR, UV, 1D, 2D NMR and HR-ESI-MS spectra data. Moreover, hexane extract and isolated compound 1 were examined for their cytotoxic activity against HeLa, T47D, and A549 cells. The results revealed the significance cytotoxic activity in the hexane extract.

Keywords: Fruit Peel, Onoceranoid-type triterpene, Meliaceae, Cytotoxicity, Anticancer, Spectroscopic Analysis

RASĀYAN J. Chem., Vol. 14, No.2, 2021
\end{abstract}

\section{INTRODUCTION}

Langsat fruit (Lansium domesticum Corr., family Meliaceae) is one of Southeast Asia's most common fruits, particularly in Indonesia. It is widely found in other Asian countries, including Malaysia, Vietnam, Cambodia, Myanmar, and the Philippines. Phytochemical analysis revealed that triterpenoids especially onoceranoids with rare and unique skeleton, onoceranoid glycosides, tetranortriterpenoid, and cycloartenoidtriterpenes are the major components in this plant. The extract and its major components from genus Lansium have shown significant biological activities such as antifeedant, anticancer, antimalarial, antidiabetic, antioxidant and antifungal. ${ }^{1-17}$

Our investigation on the hexane soluble portion of methanol fruits peel extract of Lansium domesticum Led to the isolation of an onoceradienedione, namely an 8,14-secogammacera-7,14(27)-dien-3,21-dione (Fig.1) Moreover, cytotoxic activity of hexane extract and the isolated compound was evaluated using MTT and XTT assay against cervical (HeLa), breast (T47D) and lung (A549) cancer cell lines in vitro.

\section{EXPERIMENTAL}

Plant Materials

Lansium domesticum Corr. fruits have been brought from markets Nganjuk, East Java, Indonesia in December 2018. The sample was cleaned and dried. Fruit peels of Lansium domesticum were cut into small 
RASĀYAN J. Chem.

Vol. 14 | No. 2 |1336-1340| April - June | 2021

parts and powder. The plant sample was identified by Herbarium Bogoriense, Biology Research Center, Cibinong, Indonesia.

\section{Extraction and Isolation}

The powder of Lansium domesticum ( $4000 \mathrm{~g}$ ) was extracted by methanol at room temperature for 3 days (3 $\times 2 \mathrm{~L}$ ) and filtered. Then, the combined filtrates were evaporated and partitioned between hexane and DCM (dichloromethane). The hexane soluble fractions $(300 \mathrm{~g})$ were then separated by VLC (vacuum liquid chromatography) using step gradient solvent mixtures of hexane-DCM to give five fractions (A-E). Fraction $\mathrm{C}(0.612 \mathrm{~g})$ was then separated by using silica gel column chromatography and eluted with the solvent mixtures of hexane-EtOAc to produce six sub-fractions $\left(\mathrm{L}_{1}-\mathrm{L}_{6}\right)$. Sub-fraction $\mathrm{L}_{3}(174 \mathrm{mg})$ has been applied to the column chromatography using the hexane-EtOAc solvent mixtures to get compound $\mathbf{1}(5.1 \mathrm{mg})$.

\section{General Procedure}

NMR spectra were measured on Bruker AVANCE III spectrometer using $\mathrm{CDCl}_{3}$ as a solvent.HR-EI-MS was obtained on a JEOL JMS-700 spectrometer. IR spectrum was determined on a FTIR-8400S (Shimadzu) spectrophotometer, where UV spectrum was recorded in $\mathrm{MeOH}$ on UV-Vis Shimadzu spectrometer. The melting point was measured on a Fisher-Johns Melting Point Apparatus. Silica gel 60 (700-200 mesh ASTM) was used for column chromatography. Analytical TLC was conducted on a pre-coated silica gel 60 $\mathrm{F}_{254}$ (Merck).

\section{Detection Method \\ Bioassay \\ Cell Culture}

Three cancer celllines, Cervical (HeLa), breast (T47D) and lung (A459) obtained from American Type Culture Collection (ATCC). Cells were cultured at $37^{\circ} \mathrm{C}$ and $5 \% \mathrm{CO}_{2}$ for 24 hours and $100 \%$ humidified in medium supplemented with $10 \%$ FBS, $1 \%$, L-glutamine and $1 \%$ penicillin/streptomycin.

\section{MTT Assay}

Cytotoxicity of test samples was used by the established method ${ }^{18}$. Briefly, cells were seeded in 96 well plates $\left(10^{4}-10^{5} \mathrm{cells} /\right.$ well $)$ and incubated in $\mathrm{CO}_{2}$ incubator at $37^{\circ} \mathrm{C}$ for 24 hours. Following incubation, cells were served with specific assay concentrations $(1.5625-100 \mu \mathrm{g} / \mathrm{mL})$ at $37^{\circ} \mathrm{C}$ in the $\mathrm{CO}_{2}$ incubator for 24 hours. Doxorubicin was used as a positive control. After incubation, a hundred $\mu \mathrm{L}$ of MTT reagent in phosphate-buffered saline $(5 \mathrm{mg} / \mathrm{mL}$ in PBS) was transferred into every well, and the plates re-incubated for 3 hours at $37{ }^{\circ} \mathrm{C}$. Then, the medium was removed, and the purple formazan was solvated in $0.1 \mathrm{~N} \mathrm{HCl}$. The absorbance was recorded on ELISA microplate reader at wavelength $560 \mathrm{~nm}$. Each experiment was carried out in triplicates. The $\%$ of cell viability was calculated by the following equation :

$$
\% \text { Cell viability }=\frac{(a-c)}{(b-c)} \times 100 \%
$$

Where $\mathrm{a}=$ absorbance of the sample, $\mathrm{b}=$ absorbance of cells control and $\mathrm{c}=$ absorbance of media control.

\section{XTT Assay}

XTT assay of extract and isolated compound were done according to the established protocol. ${ }^{18}$

\section{RESULTS AND DISCUSSION}

Compound $\mathbf{1}$ was isolated as white needle-like crystals, and the molecular formula $\mathrm{C}_{30} \mathrm{H}_{46} \mathrm{O}_{2}$ was determined by HREI-MS ( $\mathrm{m} / \mathrm{z}$ 438.3498, [M+H] ${ }^{+}$(Calcd. 438.6960), ${ }^{1} \mathrm{H}$ NMR, and ${ }^{13} \mathrm{C}$ NMR spectra data (Table-1). The UV spectrum of compound 1 in $\mathrm{MeOH}$ displayed absorption maxima at $214.5 \mathrm{~nm}$. The presence of carbonyl and olefinic groups were suggested by IR spectra data. The proton NMR $(600 \mathrm{MHz}$, $\left.\mathrm{CDCl}_{3}\right)$ displayed seven methyl singlet signals at $\delta_{\mathrm{H}} 0.84\left(\mathrm{H}_{3}-28\right), 0.94\left(\mathrm{H}_{3}-25\right), 1.01\left(\mathrm{H}_{3}-30\right), 1.04\left(\mathrm{H}_{3}-23\right)$, $1.08\left(\mathrm{H}_{3}-24\right), 1.10\left(\mathrm{H}_{3}-29\right)$, and $1.72\left(\mathrm{H}_{3}-26\right)$; eight multiplet methylene signals at $\delta_{\mathrm{H}} 1.42,2.06\left(\mathrm{H}_{2}-1\right), 2.24$, $2.70\left(\mathrm{H}_{2}-2\right), 1.92,2.40\left(\mathrm{H}_{2}-11\right), 1.92,2.40\left(\mathrm{H}_{2}-12\right), 2.42\left(\mathrm{H}_{2}-15\right), 1.67,1.49\left(\mathrm{H}_{2}-16\right), 1.54,2.00\left(\mathrm{H}_{2}-19\right)$, 
RASĀYAN J. Chem.

Vol. 14 | No. 2 |1336-1340| April - June | 2021

and 2.21, $2.61\left(\mathrm{H}_{2}-20\right)$; one doublet of doublet methylene signals at $\delta_{\mathrm{H}} 1.35,1.26\left(\mathrm{H}_{2}-6\right)$; an olefinic broad singlet signal at $\delta_{\mathrm{H}} 5.42(\mathrm{H}-7)$; three multiplet methine signals at $\delta_{\mathrm{H}} 1.58(\mathrm{H}-17), 1.60(\mathrm{H}-13)$, and $1.65(\mathrm{H}-$ 9); and the remaining one set of olefinic singlet methylene signals 4.92, $4.61\left(\mathrm{H}_{2}-27\right)$. The ${ }^{13} \mathrm{C} \mathrm{NMR}(150$ $\mathrm{MHz}$ ) revealed the presence of seven methyl carbons signals at $\delta_{\mathrm{C}} 13.5$ (C-25), 14.2 (C-28), 21.6 (C-30), 22.0 (C-24), 22.2 (C-26), $25.0(\mathrm{C}-23)$, and 26.1(C-29); ten methylene carbon signals ( $9 \mathrm{sp}^{3}$ and $\left.1 \mathrm{sp}^{2}\right)$ at $\delta_{\mathrm{C}}$ 24.0 (C-11), 24.1 (C-12), 25.2 (C-16), 25.3 (C-6), 34.6 (C-2), 34.7 (C-20), 37.8 (C-19), 37.9 (C-15), 38.1 $(\mathrm{C}-1)$, and 107.7 (C-27); five methine carbon signals (including $4 \mathrm{sp}^{3}$ and $\left.1 \mathrm{sp}^{2}\right)$ at $\delta_{\mathrm{C}} 51.6(\mathrm{C}-5), 54.3(\mathrm{C}-$ 13), 55.2 (C-17), 56.0 (C-9), and 121.8 (C-7); eight quaternary carbon signals (including 2 carbonyl, $2 \mathrm{sp}^{2}$, $\left.4 \mathrm{sp}^{3}\right)$ at $\delta_{\mathrm{C}} 14.3$ (C-14), 39.2 (C-18), 36.5 (C-10), 47.4 (C-4 and C-22), 135.4 (C-8), $216.6(\mathrm{C}-21)$, and 216.8 (C-3). According to the above spectra data, compound 1 has an onoceranoid-type triterpene. The structure of compound 1 was further confirmed by COSY and HMBC correlations (Fig.-2). Compound 1 was then established by comparison with the reporter data as an onoceradienedione (Fig.-1). ${ }^{19}$

Moreover, cytotoxicity of hexane extracts and compound 1 were evaluated against HeLa, T47D and A549 cell lines. The results are shown in Table-2. According to the Cancer Institute standards, crude extracts with $\mathrm{IC}_{50}$ values of $<20 \mu \mathrm{g} / \mathrm{mL}$, and a natural compound that has $\mathrm{IC}_{50}$ values of $<4$ or $10 \mu \mathrm{g} / \mathrm{mL}$ are considered active $^{20}$. Therefore, hexane extract displayed potential cytotoxic activity against T47D $\left(\mathrm{IC}_{50} 0.10 \mu \mathrm{g} / \mathrm{mL}\right)$ and A549 ( $\left.\mathrm{IC}_{50} 18.83 \mu \mathrm{g} / \mathrm{mL}\right)$ cell lines, but compound $\mathbf{1}$ demonstrated weak activity against only A549 $\left(\mathrm{IC}_{50} 13.71 \mu \mathrm{g} / \mathrm{mL}\right)$ cell lines.

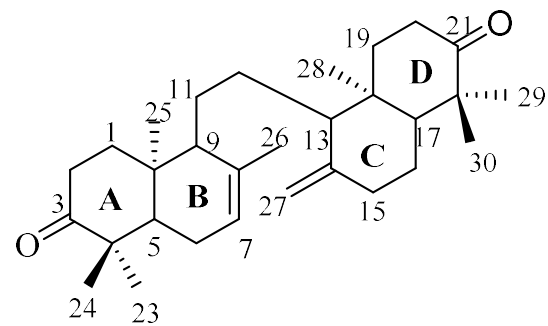

Fig.-1: Chemical Structure of Compound 1 from the Fruit Peel of Lansium domesticum

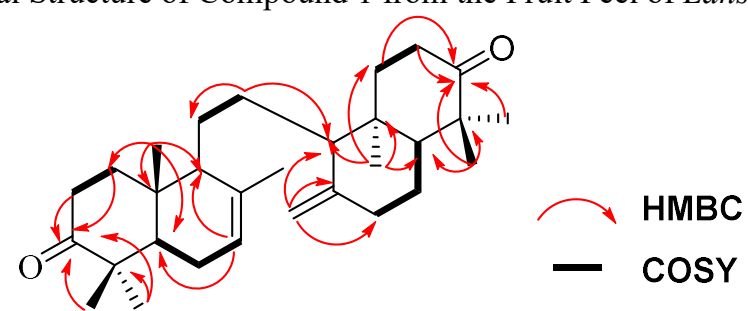

Fig.-2: HMBC and COSY Experiments of Compound 1

Table-1: NMR Spectral Data of Compound 1

\begin{tabular}{c|c|c|c}
\hline Position & $\delta_{\mathrm{H}}(\mathrm{ppm})(\mathrm{mult}, J$ in $\mathrm{Hz})$ & $\delta_{\mathrm{C}}(\mathrm{ppm})$ & HMBC \\
\hline \multirow{2}{*}{1} & $1.42(1 \mathrm{H}, m)$ & 38.1 & \multirow{2}{*}{ C-2, C-3, C-10 } \\
\cline { 2 - 3 } 2 & $2.06(1 \mathrm{H}, m)$ & 34.6 & \multirow{2}{*}{ C-1, C-3, C-10 } \\
\cline { 2 - 3 } & $2.24(1 \mathrm{H}, m)$ & 216.8 & - \\
\hline 3 & - & 47.4 & - \\
\hline 4 & - & 51.6 & $\mathrm{C}-9, \mathrm{C}-10, \mathrm{C}-24$ \\
\hline 5 & $1.59(1 \mathrm{H}, d d, J=7.0,5.0 \mathrm{~Hz})$ & 25.3 & $\mathrm{C}-7, \mathrm{C}-10$ \\
\hline \multirow{2}{*}{6} & $1.35(1 \mathrm{H}, d d, J=7.0,10.0 \mathrm{~Hz})$ & 121.8 & $\mathrm{C}-5, \mathrm{C}-9, \mathrm{C}-26$ \\
\cline { 2 - 3 } & $1.26(1 \mathrm{H}, d d, J=5.0,10.0 \mathrm{~Hz})$ & 135.4 & - \\
\hline 7 & $5.42(1 \mathrm{H}, s b r)$ & 56.0 & $\mathrm{C}-26$ \\
\hline 9 & - & 36.5 & - \\
\hline 10 & $1.65(1 \mathrm{H}, m)$ & 24.0 & $\mathrm{C}-9$ \\
\hline 11 & - & & \\
\hline
\end{tabular}

1338 
RASĀYAN J. Chem.

Vol. 14 | No. 2 |1336-1340| April - June | 2021

\begin{tabular}{|c|c|c|c|}
\hline & $2.40(1 \mathrm{H}, m)$ & & \\
\hline \multirow{2}{*}{12} & $1.92(1 \mathrm{H}, m)$ & \multirow{2}{*}{24.1} & \multirow{2}{*}{ C-13 } \\
\hline & $2.40(1 \mathrm{H}, m)$ & & \\
\hline 13 & $1.60(1 \mathrm{H}, m)$ & 54.4 & C-28 \\
\hline 14 & - & 147.3 & - \\
\hline 15 & $2.42(2 \mathrm{H}, m)$ & 37.9 & C-14, C-16, C-27 \\
\hline \multirow{2}{*}{16} & $1.67(1 \mathrm{H}, m)$ & \multirow{2}{*}{25.2} & \multirow{2}{*}{ C-17 } \\
\hline & $1.49(1 \mathrm{H}, m)$ & & \\
\hline 17 & $1.58(1 \mathrm{H}, m)$ & 55.2 & C-13, C-18; C-29 \\
\hline 18 & - & 39.2 & - \\
\hline \multirow{2}{*}{19} & $1.54(1 \mathrm{H}, m)$ & \multirow{2}{*}{37.8} & \multirow{2}{*}{ C-18, C-20, C-21 } \\
\hline & $2.00(1 \mathrm{H}, m)$ & & \\
\hline \multirow{2}{*}{20} & $2.21(1 \mathrm{H}, m)$ & \multirow{2}{*}{34.7} & \multirow{2}{*}{ C-18, C-19, C-21 } \\
\hline & $2.61(1 \mathrm{H}, m)$ & & \\
\hline 21 & - & 216.6 & - \\
\hline 22 & - & 47.7 & - \\
\hline 23 & $1.04(3 \mathrm{H}, s)$ & 25.0 & $\mathrm{C}-3, \mathrm{C}-4, \mathrm{C}-5$ \\
\hline 24 & $1.08(3 \mathrm{H}, s)$ & 22.0 & $\mathrm{C}-3, \mathrm{C}-4, \mathrm{C}-5$ \\
\hline 25 & $0.94(3 \mathrm{H}, s)$ & 13.5 & C-1, C-5, C-9, C-10 \\
\hline 26 & $1.72(3 \mathrm{H}, s)$ & 22.2 & C-7, C-8, C-9 \\
\hline \multirow{2}{*}{27} & $4.92(1 \mathrm{H}, s)$ & \multirow{2}{*}{107.7} & \multirow{2}{*}{$\mathrm{C}-13, \mathrm{C}-14, \mathrm{C}-15$} \\
\hline & $4.61(1 \mathrm{H}, s)$ & & \\
\hline 28 & $0.84(3 \mathrm{H}, s)$ & 14.2 & C-13, C-17, C-18, C-19 \\
\hline 29 & $1.10(3 \mathrm{H}, s)$ & 26.1 & $\mathrm{C}-17, \mathrm{C}-21, \mathrm{C}-22$ \\
\hline 30 & $1.01(3 \mathrm{H}, s)$ & 21.6 & $\mathrm{C}-17, \mathrm{C}-21, \mathrm{C}-22$ \\
\hline
\end{tabular}

$*$ Recorded at $600 \mathrm{MHz}$ for ${ }^{1} \mathrm{H}$ and $150 \mathrm{MHz}$ for ${ }^{13} \mathrm{C}-\mathrm{NMR}$.

Table-2: The $\mathrm{IC}_{50} \pm$ S.D. $(\mu \mathrm{g} / \mathrm{ml})$ of the Compound and Crude Extract from the Peel of Lansium domesticum and Doxorubicin

\begin{tabular}{c|l|c|c|c}
\hline \multirow{2}{*}{ No. } & \multicolumn{1}{|c|}{ Compound } & \multicolumn{3}{c}{$\mathrm{IC}_{50} \pm$ S.D. $(\mu \mathrm{g} / \mathrm{ml})$} \\
\cline { 3 - 5 } & \multicolumn{1}{|c}{ HeLa } & \multicolumn{1}{c}{ T47D } & \multicolumn{1}{c}{ A549 } \\
\hline 1 & $\begin{array}{l}\text { 8,14-Secogammacera-7,14(27)-dien-3,21- } \\
\text { dione (onoceradienedione) }\end{array}$ & $32.39 \pm 1.17$ & $30.69 \pm 1.87$ & $13.71 \pm 0.42$ \\
\hline 2 & $n$-Hexane extract & $59.55 \pm 4.29$ & $0.10 \pm 5.88$ & $18.83 \pm 2.58$ \\
\hline 3 & Doxorubicin & $2.83 \pm 0.18$ & $0.04 \pm 0.03$ & - \\
\hline
\end{tabular}

* All data represent the mean of $\mathrm{IC}_{50} \pm$ S.D. of triplicates.

\section{CONCLUSION}

In conclusion, onoceradienedione 1 was produced from the hexane soluble portion of methanol fruits peel extract of Lansium domesticum. The structure of the isolated compound was done by advance spectroscopic techniques, including IR, UV, NMR, and HR-ESI-MS spectra data. Moreover, cytotoxicity of hexane extract and the isolated compound was evaluated using MTT and XTT assay against HeLa, T47D, and A459 cell lines. The results showed that hexane extract displayed significant cytotoxic activity against T47D and A459 cell lines, but the isolated compound has weak activity against A459 cell lines.

\section{ACKNOWLEDGEMENT}

The author expresses high appreciation to: (1) Institute for Research and Community Services, UniversitasAirlangga, Surabaya, Indonesia for "HIBAH RISET MANDAT KOLABORASI MITRA LUAR NEGERI" funding support. (2) Faculty of Pharmacy, Meijo University, Nagoya, Japan for NMR data. (3) Cancer Research Lab, Institute of Biosciences, Universiti Putra Malaysia, 43400 UPM Serdang, Selangor, Malaysia for the cytotoxic analysis.

\section{REFERENCES}

1. K. Venkatachalam, Fruits, 71(5), 289(2016), DOI:10.1051/fruits/2016026 
RASĀYAN J. Chem.

Vol. 14 | No. 2 |1336-1340| April - June | 2021

2. Rudiyansyah, A.H Alimudin, Masriani, R. Muharini, P. Proksch P, Phytochem Letters, 23, 90(2018), DOI:10.1016/j.phytol.2017.11.019

3. M. Nishizawa, M. Emura, H. Yamada, M. Shiro, Chairul, Y. Hayashi, H. TokudaV, Tetrahedron Letters, 30(41), 5615(1989), DOI:10.1016/S0040-4039(01)93813-4

4. T. Tanaka, M. Ishibashi, H. Fujimoto, E. Okuyama, T. Koyano, T. Kowithayakorn, M Hayashi, K. Komiyama, Journal of Natural Products, 65(11), 1709(2002), DOI:10.1021/np020239o

5. S. Omar, M. Marcotte, P. Fields, P.E. Sanchez, L. Poveda, R. Mata, A. Jimenez, T. Durst, J. Zhang, S. MacKinnon, D. Leaman, J.T. Arnason, B.J.R. Philogene, Journal of Stored Product Research, 43, 92(2007), DOI:10.1016/j.jspr.2005.11.005

6. S.H Dong, C.R Zhang, Y.W Wu, J.M Yue, Journal of Natural Products, 74(5), 1042(2011), DOI: $10.1021 / \mathrm{np} 100943 \mathrm{x}$

7. T. Mayanti, R. Tjokronegoro, U. Supratman, M.R Mukhtar, K. Awang, A.H. A. Hadi, Molecules, 16(4), 2785(2011), DOI:10.3390/molecules16042785

8. M. Nishizawa, H. Nishide, Y. Hayashi, S. Kosela, Tetrahedron Letters, 23, 1349(1982), DOI: $10.1016 /$ S0040-4039(00)87101-4

9. T. Potipiranum, W. Worawalai, P. Phuwapraisirisan, Natural Product Research, 32(16), 1881(2018), DOI: $10.1080 / 14786419.2017 .1354184$

10. M. Nishizawa, H. Nishide H, S. Kosela, Y. Hayashi Y, Journal of Organic Chemistry, 48, 4462(1983), DOI: $10.1021 /$ jo00172a004

11. R. Ramadhan, W. Worawalai, Natural Product Research, 33(20), 2917(2019), DOI: $10.1080 / 14786419.2018 .1510395$

12. T. Mastsumoto, T. Kitagawa, S. Teo, Y. Anai, R. Ikeda, D. Imahori, H.S.B. Ahmad, T. Watanabe, Journal of Natural Products, 81(10), 2187 (2018), DOI:10.1021/acs.jnatprod.8b00341

13. M. Nishizawa M, Y. Nademoto, S. Satrapradja, M. Shiro, Y. Hayashi, Journal of Organic Chemistry, 50, 5487(1985), DOI:10.1021/jo00350a009

14. M. Nishizawa, Y. Nademoto, S. Sastrapradja, M. Shiro, Y. Hayashi, Phytochemistry, 27(1), 237(1988), DOI: $10.1016 / 0031-9422(88) 80622-8$

15. N. Saewan, J.D Sutherland, K. Chantrapromma, Phytochemistry, 67(20), 2288(2006), DOI: $10.1016 /$ j.phytochem.2006.07.005

16. T. Mayanti, J. Sianturi, D. Harneti, Darwati, U. Supratman, M. Mustaqim, H.K Fun, Molbank, 2015(4), M880(2015), DOI: 10.3390/M880

17. D.T.T. Yapp and S.Y. Yap, Journal of Ethnopharmacol, 85(1), 145(2003), DOI:10.1016/s03788741(02)00375-6

18. CCRC, 2014, Prosedur Tetap Uji Sitotoksik Metode MTT, Faculty of Pharmacy Gadjah Mada University, Yogyakarta, (Text in Indonesia), http://ccrc.farmasi.ugm.ac.id/wp-content/uploads/10_sopuji-sitotoksik-metode-mtt.pdf

19. T. Mayanti, W.D Natawigena, U. Supratman, R. Tjokronegoro, International Seminar on Chemistry 2008, Bandung 30-31 October 2008, http://pustaka.unpad.ac.id/wpcontent/uploads/2010/11/antibacterial_terpenoid_from the bark_of lansium domesticum.pdf

20. A. M. Alabsi, K.L. Lim, I. C Paterson, R Ali-Saeed, B.A Muharram, BioMed Research International, 3, 1(2016), DOI:10.1155/2016/4904016

[RJC-6044/2020] 